(1)

CrossMark

\title{
Cyst-related primary lung malignancies: an important and relatively unknown imaging appearance of (early) lung cancer
}

\author{
Onno M. Mets ${ }^{1}$, Cornelia M. Schaefer-Prokop ${ }^{2,3}$ and Pim A. de Jong ${ }^{1}$
}

Affiliations: ${ }^{1}$ Dept of Radiology, University Medical Center Utrecht, Utrecht, The Netherlands. ${ }^{2}$ Diagnostic Imaging Analysis Groups, Radboud University Nijmegen Medical Centre, Nijmegen, The Netherlands. ${ }^{3}$ Dept of Radiology, Meander Medical Center, Amersfoort, The Netherlands.

Correspondence: Onno M. Mets, Dept of Radiology, University Medical Center Utrecht, Heidelberglaan 100, Huispostnummer E01.132, Postbus 85500, Utrecht 3508 GA, The Netherlands. E-mail: o.m.metsवumcutrecht.nl

@ERSpublications

Primary lung cancer can present as cystic airspace with an associated solid component, and seems to be a relatively common imaging phenotype of (early) lung cancer. This morphology is often not recognised and misinterpreted, leading to diagnostic delay. http://ow.ly/hZrJ30mIVX1

Cite this article as: Mets OM, Schaefer-Prokop CM, de Jong PA. Cyst-related primary lung malignancies: an important and relatively unknown imaging appearance of (early) lung cancer. Eur Respir Rev 2018; 27: 180079 [https://doi.org/10.1183/16000617.0079-2018].

ABSTRACT It is well known that lung cancer can manifest itself in imaging as solid and subsolid nodules or masses. However, in this era of increased computed tomography use another morphological computed tomography appearance of lung cancer is increasingly being recognised, presenting as a malignancy in relation to cystic airspaces. Despite the fact that it seems to be a relatively common finding in daily practice, literature on this entity is scarce and presumably the overall awareness is limited. This can lead to misinterpretation and delay in diagnosis and, therefore, increased awareness is urgently needed. This review aims to illustrate the imaging appearances of cyst-related primary lung malignancies, demonstrate its mimickers and potential pitfalls, and discuss the clinical implications based on the available literature and our own experience in four different hospitals.

\section{Cyst-related primary lung malignancies}

Management guidelines for indeterminate small solid pulmonary lesions are available for both clinical use and in the screening setting $[1,2]$. This has led to more (serial) computed tomography (CT) imaging and more structured and uniform interpretation and surveillance of pulmonary nodules. Over a decade ago it emerged that beside solid nodules and masses a second group of morphologically distinct lung cancers existed; subsolid pulmonary malignancies. Instead of a solid nodular lung lesion, these present as persistent pure ground-glass or part-solid pulmonary nodules. This distinction is highly relevant in daily practice as the behaviour of subsolid lesions differs significantly from that of solid lesions, being more commonly adenocarcinomas and showing slower growth but higher malignancy rates than solid nodules $[3,4]$. However, it is now increasingly recognised that primary lung cancer does not always appear as either a solid or subsolid nodule on imaging. An important and presumably under recognised morphology of (early) lung cancer is the cyst-related primary lung malignancy. Terminology used in prior literature includes "(peri)cystic adenocarcinoma" and "cancers in association with cystic airspaces". The entity has been reported on in a few smaller original studies [5-9], which were recently summarised in an imaging review paper [10]. In total, less than 100 cases have been reported, originating from both a screening and a

Provenance: Submitted article, peer reviewed.

Received: Aug 152018 | Accepted after revision: Nov 112018

Copyright CERS 2018. ERR articles are open access and distributed under the terms of the Creative Commons Attribution Non-Commercial Licence 4.0 
clinical setting. This review now adds multiple cases of cyst-related primary lung malignancies originating from routine care in four separate hospitals.

Exact numbers on incidence of primary lung cancers presenting as cyst-related lesions are unknown, but estimates range between $0.5 \%$ and $4 \%[5,7,8]$. We and others $[6,7]$ have found several cases where lesions eventually progress into solid nodules or masses, and it is therefore likely that the incidence of this entity is in fact underestimated and either not always imaged or recognised at an early stage, as illustrated in figure 1.

\section{CT acquisition and imaging appearances of cyst-related primary lung malignancies}

In general, to optimally assess lung lesion morphology with CT it is important to use all three orthogonal planes and thin-slice reconstructions (slice thickness $\leqslant 1.5 \mathrm{~mm}$ ), as thicker slices will increase volume averaging and loss of details. Also, dose reduction is important in serial imaging $[1,2]$.

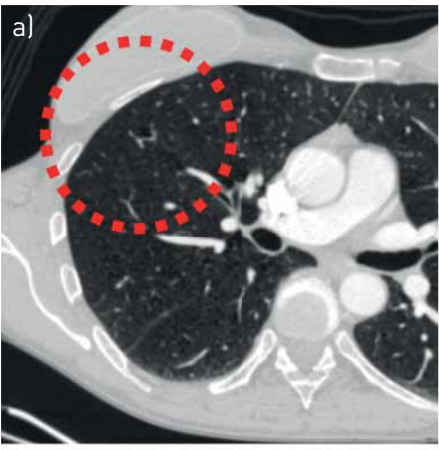

d)

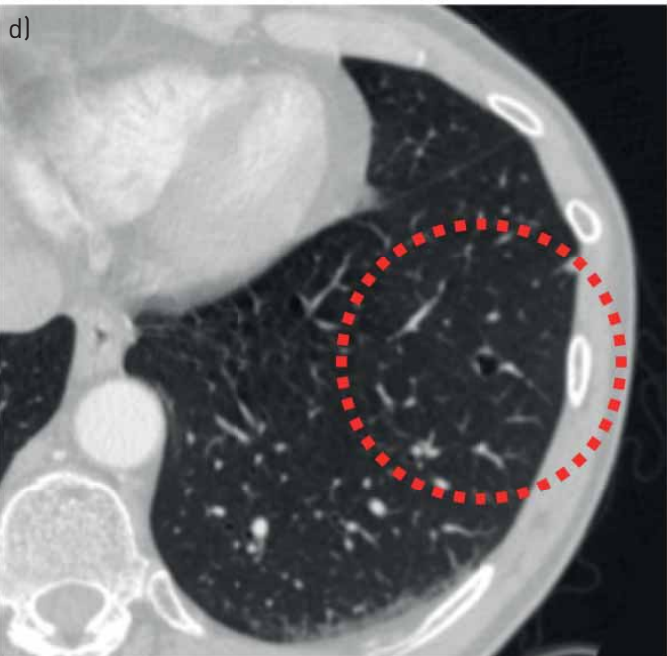

f)

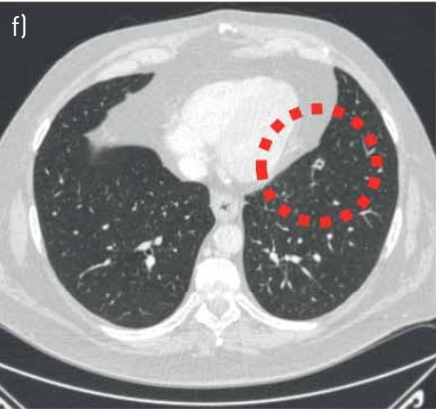

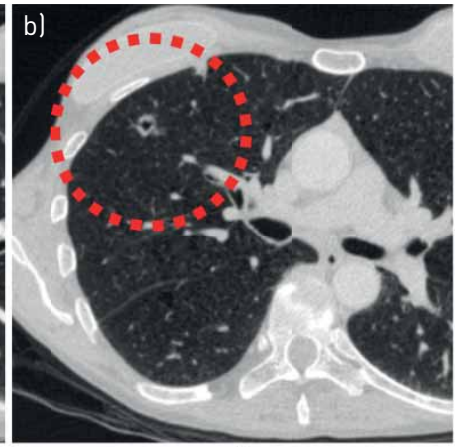

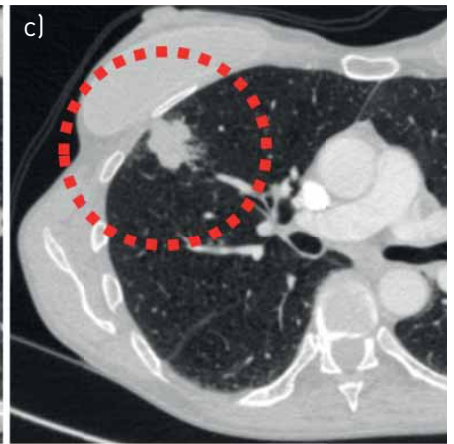

e)
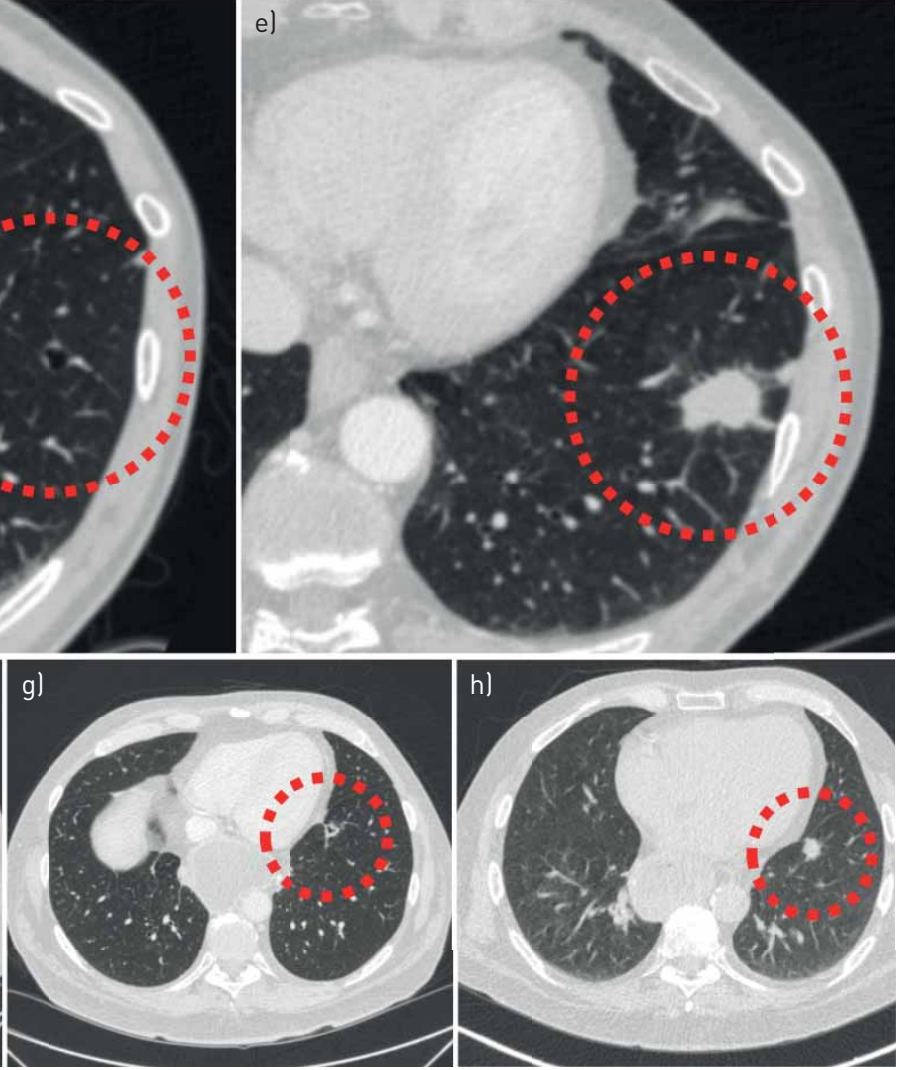

FIGURE 1 Cyst-related lung malignancies might be more common than expected, as the early manifestations of solid masses may not always be captured. a-c) Baseline and follow-up computed tomography (CT) scans in a 54-year-old female retrospectively show a cystic airspace with asymmetrical wall thickening, as a precursor of a solid mass 3 years later. d, e) Baseline CT scans in a 57-year-old male retrospectively show a cystic airspace with asymmetrical wall thickening, as a precursor of a solid mass 4 years later. $\mathrm{f}-\mathrm{h}$ ) Baseline and follow-up CT scans in a 60-year-old male with oesophageal carcinoma retrospectively show a thick-walled cystic lesion, as a precursor of a solid mass 2 years later. All three cyst-related lesions were not acknowledged as being suspicious. Potentially, these three adenocarcinomas could have been diagnosed at a lower T-stadium. 

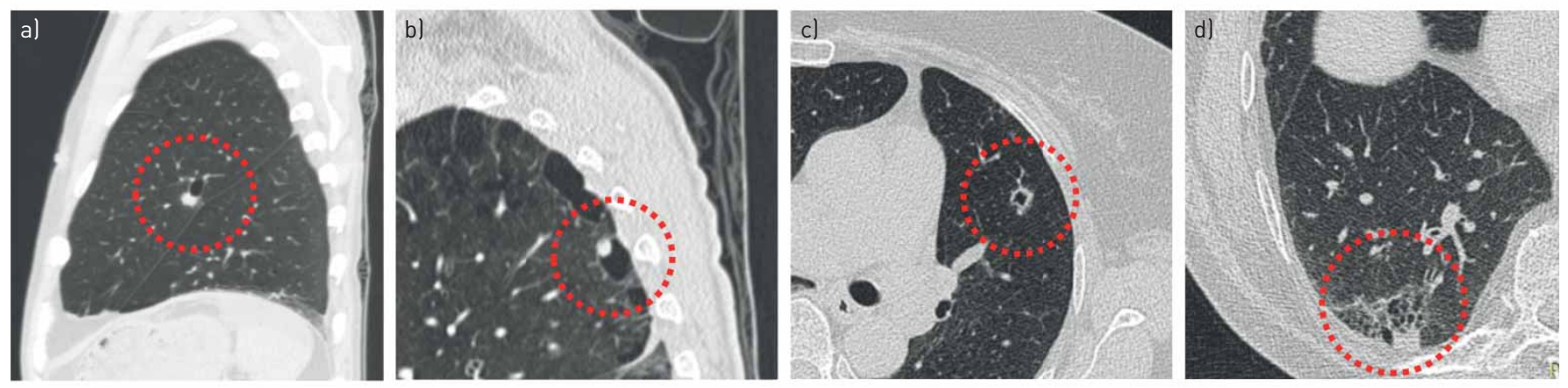

FIGURE 2 Morphological subtypes of cyst-related primary lung malignancies. a) Type 1: cyst-related malignancy with a nodular exophytic solid component. b) Type 2: cyst-related malignancy with a nodular endophytic solid component. c) Type 3: cyst-related malignancy with a circumferential wall thickening. d) Type 4: multilocular cyst-related malignancy.

Prior studies have proposed a classification of four subtypes of cyst-related lung malignancies, based on the location and morphology of the solid component in relation to the cystic area [6,11]. Type 1 and 2 are described as a cystic airspace with an exophytic or endophytic solid nodule, respectively; type 3 are described as a cystic airspace with an asymmetrical or circumferential wall thickening; and type 4 are described as a multilocular cystic lesion with interposed solid tissue. Figure 2 provides examples of these four cyst-related primary lung malignancy subtypes. MASCALCHI et al. [6] added type 4 as a separate imaging subtype to the original classification of MAKI et al. [11]. This subtype seems to be more often associated with some ground-glass components and may be somewhere on the interface between solid and subsolid cyst-related lung cancers. Although the four proposed imaging subtypes are morphologically distinctly different from each other, the differences with respect to growth rate, biological behaviour and prognosis are currently not known as overall reported numbers are low and prospective studies and longitudinal data are lacking. Moreover, there may be unclassifiable lesions (figure 3) and classification is probably prone to interobserver variability (figure 4). Also, over time, a single lesion may turn from one imaging subtype into another [6,7]. At this time there seems no role for the classification system in daily clinical practice; although the usefulness of the imaging classification in daily practice is unknown, it is nevertheless of utmost importance to acknowledge the entity of cyst-related lung malignancies as a whole and recognise all the different imaging appearances. This applies not only to radiologists, but also to pulmonologists, nuclear medicine physicians and other clinicians. Misinterpretation can lead to withholding of (long-term) follow-up imaging and will result in diagnostic and therapeutic delay (figures 1 and 5).
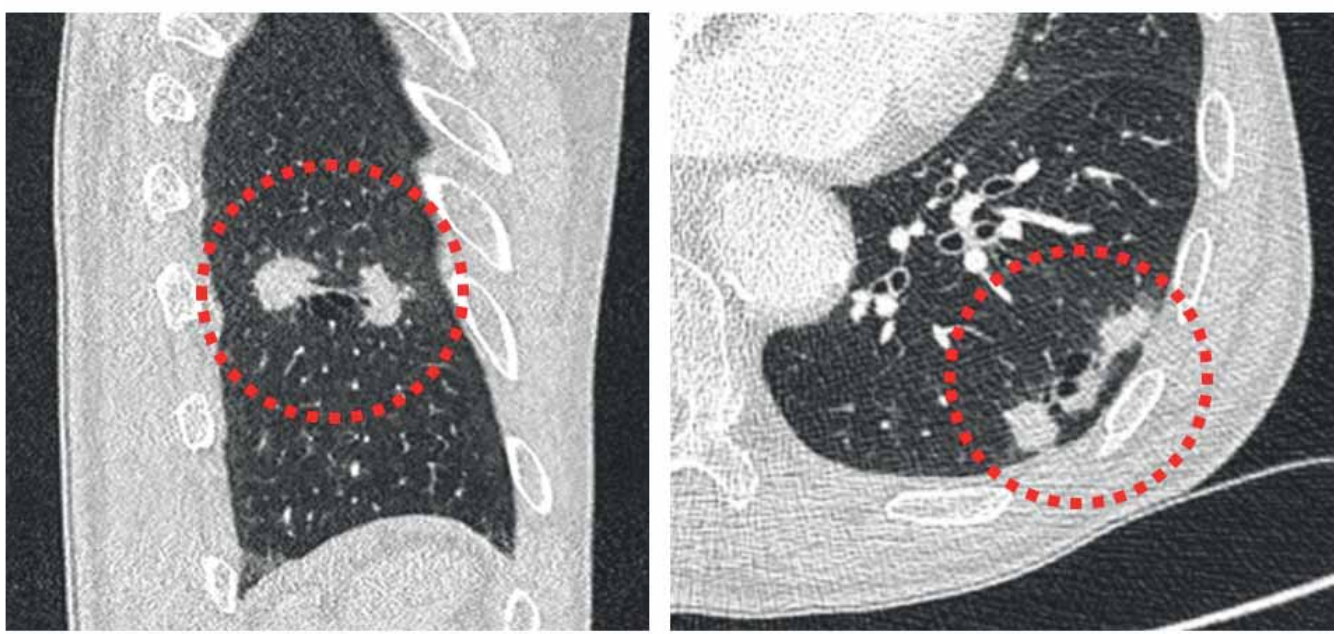

FIGURE 3 Cyst-related primary lung malignancies do not always conform to a specific subtype. A chest radiograph in an 82-year-old male showed an incidental finding, which on computed tomography imaging proved to be a cyst-related lung lesion. The lesion does not easily conform to one of the suggested subtypes as it is more bipartite. Flurodeoxyglucose-positron emission tomography computed tomography showed activity and growth was observed over 6 months despite antibiotic therapy. Stereotactic radiotherapy was performed without pathological confirmation of malignancy. 

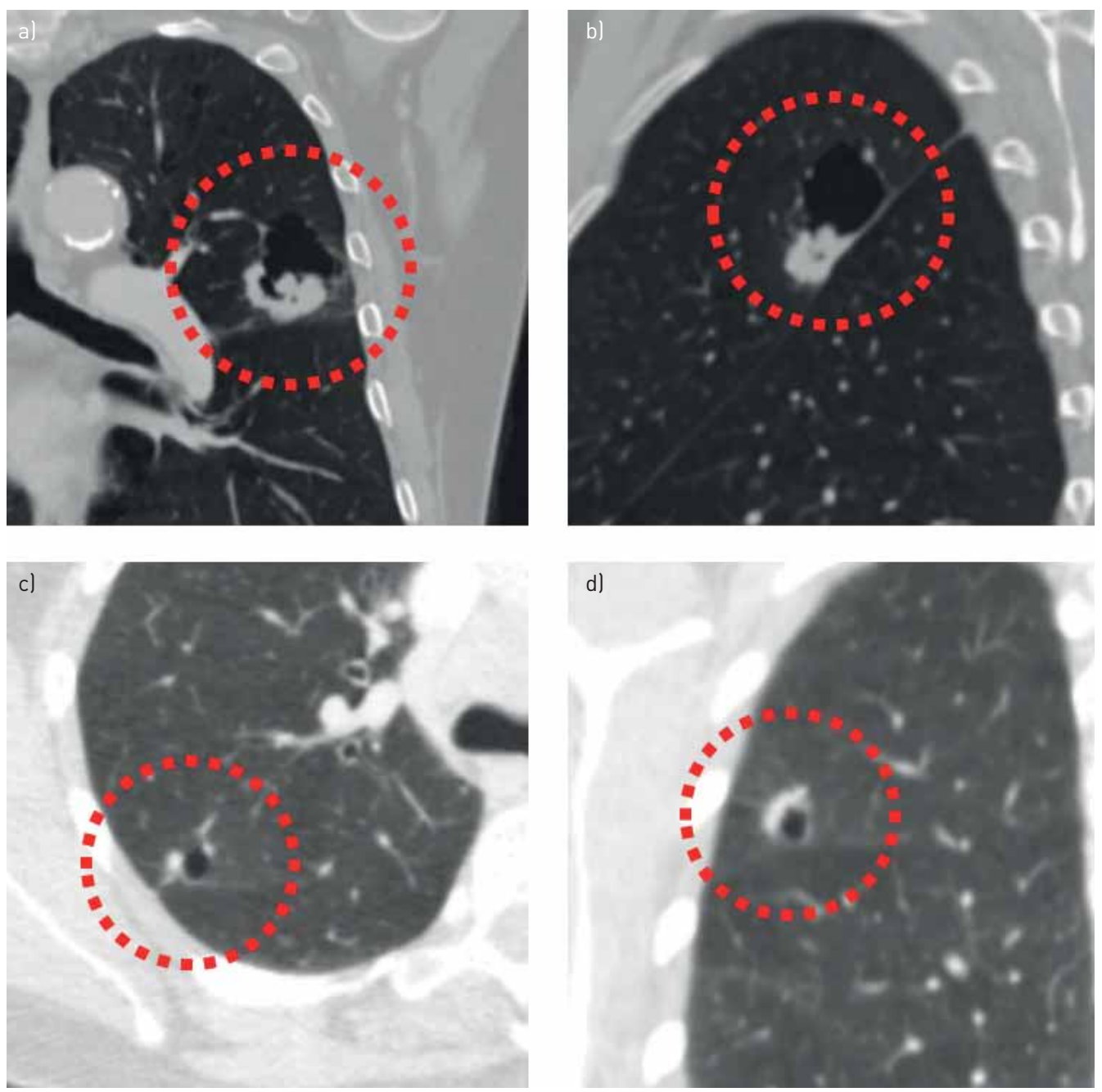

FIGURE 4 Classification of cyst-related primary lung malignancies may be prone to interobserver variation. $a, b)$ Cyst-related lung cancer in a 74-year-old male proved to be squamous cell carcinoma after resection. Morphologically, it might be characterised as a type 2 lesion due to its endophytic solid component (a), as a type 1 lesion due to its exophytic solid component more caudally (b), or maybe as a type 3 lesion due to the asymmetrical wall thickening (a). c, d) Cyst-related lung cancer in a 60-year-old female with synchronous small cell lung carcinoma in the left upper lobe. This lesion was not acknowledged as a suspect finding. It might be characterised as a type 1 lesion due to exophytic solid components (c), as unclassifiable because of two separate exophytic solid components (c), or maybe as a type 3 lesion because the most cranial solid component in a coronal plane shows more asymmetrical wall thickening (d).

In our experience, type 1 and 2 are generally more often recognised as being suspect because they involve a focal solid nodule. On the contrary, type 3 and 4 lesions may be more prone to misinterpretation given their atypical imaging appearance with (circumferential) cyst-wall thickening and a multilocular aspect, respectively. This represents morphology that is distinctly different from what typical lung cancers look like. Importantly, we found that the type 3 lesions with asymmetrical or circumferential wall thickening are most often encountered (figure 6), although exact numbers on subtype incidence require further (prospective) studies.

\section{Pathogenesis and pathology}

There are varying theories on the pathogenesis of cyst-related malignancies, and it is doubtful whether there is a single underlying mechanism in all lesions. FAROoQI et al. [5] did not find histopathological evidence for congenital cystic abnormalities in their cases or signs of other cystic lung diseases. In our experience and that of others [5, 6] most cases that provide old CT imaging do show a pre-existing cystic airspace before solid components develop. Thus, it remains undecided as to whether there is a pre-existing cystic airspace in which a malignancy develops, or whether a cyst is formed by a check-valve mechanism due to a microscopically small malignancy that only becomes visible after substantial growth. 

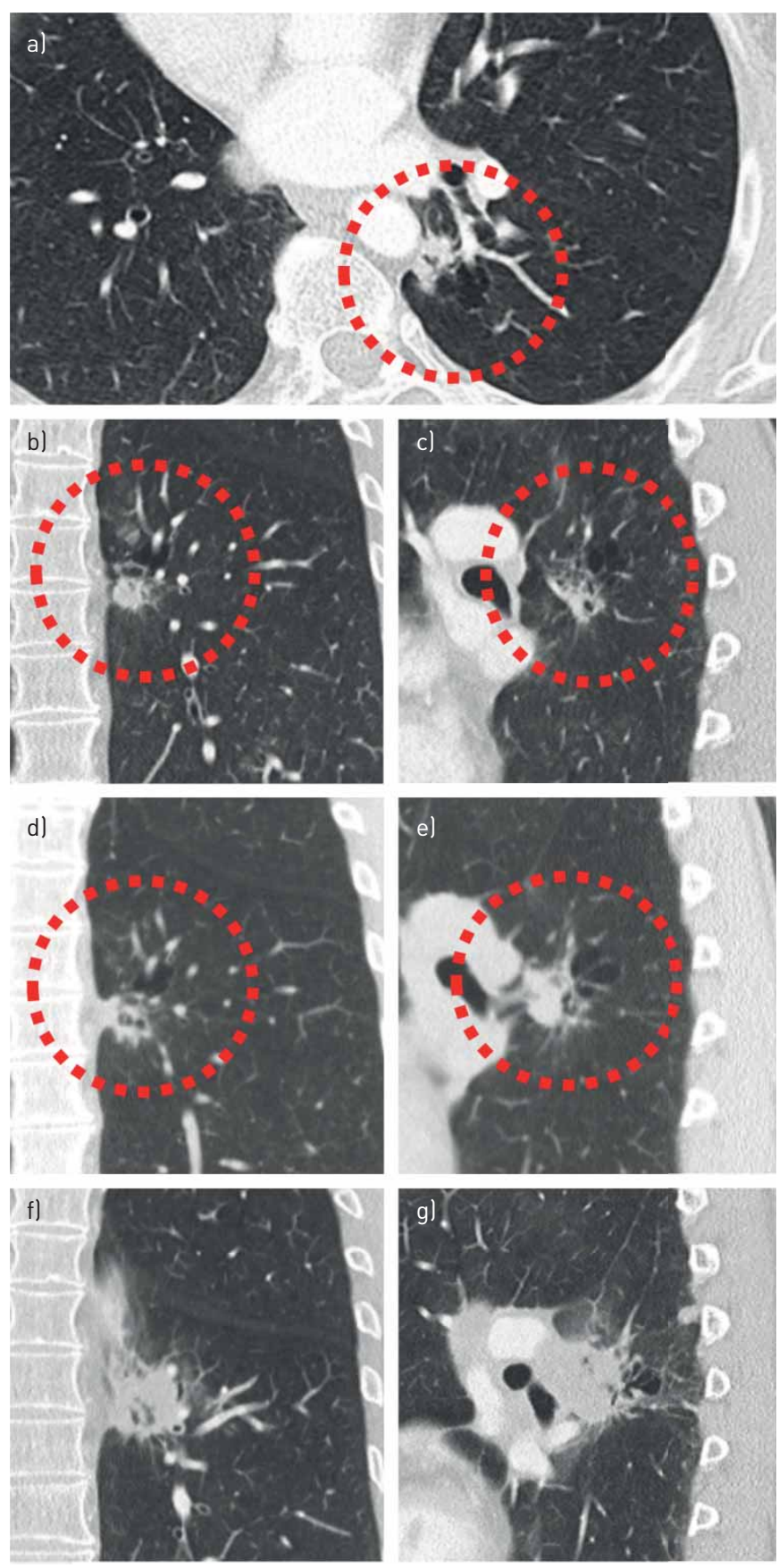

FIGURE 5 Failure to recognise cyst-related malignancies can lead to significant diagnostic delay. a-c) A cyst-related lesion in the left lower lobe of a 57-year-old female without a relevant history, shown in three orthogonal planes. Initially the lesion was interpreted to represent smoking-related changes in an emphysematous lung with several small nodular consolidations. A flurodeoxyglucose-positron emission tomography computed tomography obtained 6 weeks later (not shown) reported slight activity, most likely due to infectious origin. $d$, e) A follow-up computed tomography (CT) scan 9 months later showed an increase of the solid component, however, this was not acknowledged. The lesion was reported to be unchanged and irrelevant. 8 months later a CT scan was obtained after an ultrasound-guided biopsy of a progressive swelling in the neck showed metastatic disease. f, gl This CT scan showed an extensive increase in lesion size and confluent left hilar lymphadenopathy. In addition, mediastinal and supraclavicular lymphadenopathy, as well as abdominal and brain metastases, were present by that time (not shown).

This check-valve mechanism is described by several authors as a possible mechanism in at least some of the cases [5-7], due to the observed increase in cystic airspace size over time (figure 7). However, this is mostly after solid components emerged that in turn increase or envelope existing cystic airspaces [10]. Next to the check-valve mechanism it has been shown that (lepidic) growth along emphysematous and 

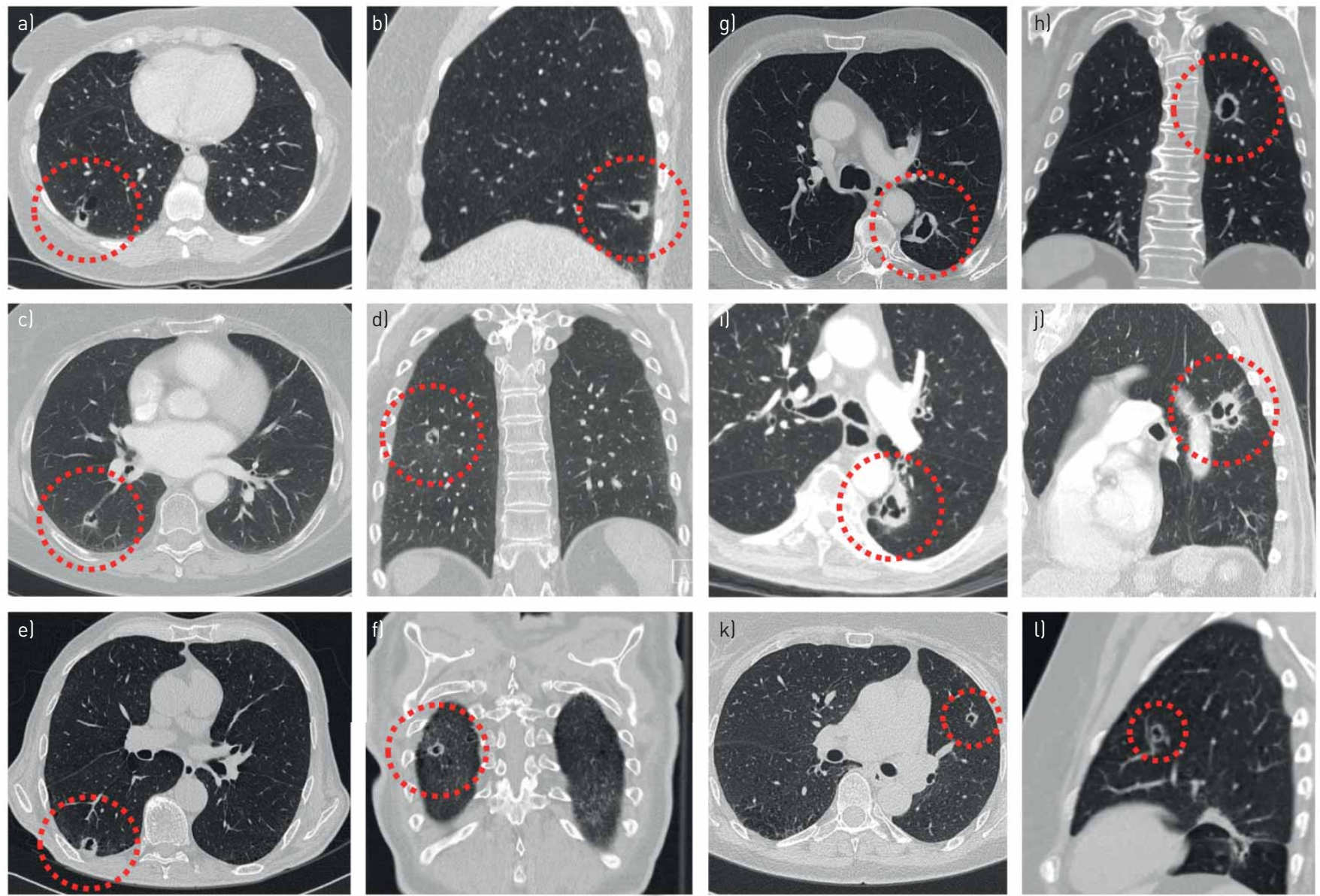

FIGURE 6 Multiple (type 3) cyst-related primary lung malignancies presenting as cystic airspaces with asymmetrical or circumferential wall thickening. a, b) A 52-year-old female with a biopsy-proven second primary adenocarcinoma in the right lower lobe, synchronous with a T1cN0M1b adenocarcinoma in the left upper lobe (not shown). c, d) A 68-year-old female with a history of breast carcinoma and left upper lobe small cell lung carcinoma. The cyst-related lesion in the right lower lobe was present at baseline and repeatedly misinterpreted as a cavitating contralateral metastasis. After 2 years of continuous increase in wall thickness pathology was obtained and showed a second primary adenocarcinoma. e, f) A 71-year-old male with a history of bladder carcinoma. Cyst-related lesion with progressive circumferential wall thickening on serial computed tomography imaging. The patient opted for stereotactic radiotherapy (STR) without obtaining pathology. g, h) A 78-year-old male without prior malignancy who presented with liver metastasis. This proved to be a moderately differentiated mucinous adenocarcinoma, probably from the lung. Work-up showed this cyst-related lung cancer in the left lower lobe. i, j) An 89-year-old male without a relevant history. Cyst-related lesion in the left lower lobe which showed slight positron emission tomography activity and increase in wall thickness over a period of 3 months. All cultures were negative. STR was performed without obtaining pathology. k, l) A 62-year-old female with a history of an adenocarcinoma in situ in the left upper lobe, treated with radiotherapy. During follow-up this cyst-related lesion developed in the left upper lobe. After consecutive growth was established over a period of 6 months it was treated with STR without obtaining pathology.

bullous walls is also possible [7]. It is unknown whether both the visible solid components, as well as the cyst wall, contain malignant cells; however, one should always resect the whole cyst-related lesion with a free margin to prevent local recurrence, as illustrated in figure 7.

Whatever the pathogenesis may be, it is important to emphasise that cyst-related malignancies are not, as often misnamed in reports, cavitating lesions. None of the prior reported cases showed signs of necrosis in the pathology specimens $[5,7]$. This is underlined by the fact that cyst-related malignancies show an in increase in wall thickness and solidification over time, while cavitation is a process where an initially solid lesion shows progressive lucency due to cell loss.

From the limited data available at this time [5-8], it is apparent that cyst-related malignancies are primary lung malignancies. They seem to mostly represent adenocarcinomas, although squamous cell carcinomas and to a lesser extent small cell carcinomas are also present. They often co-exist with pulmonary emphysema and are mainly found in current or former smokers. No lobe predominance appears present; however, they seem to be located more often peripherally than centrally. Our cases are in line with this data. Also, prior studies have mentioned a surprisingly high number of subjects (42-47\%) where cyst-related malignancies are metachronous or synchronous second primary lung malignancies $[6,7]$. 

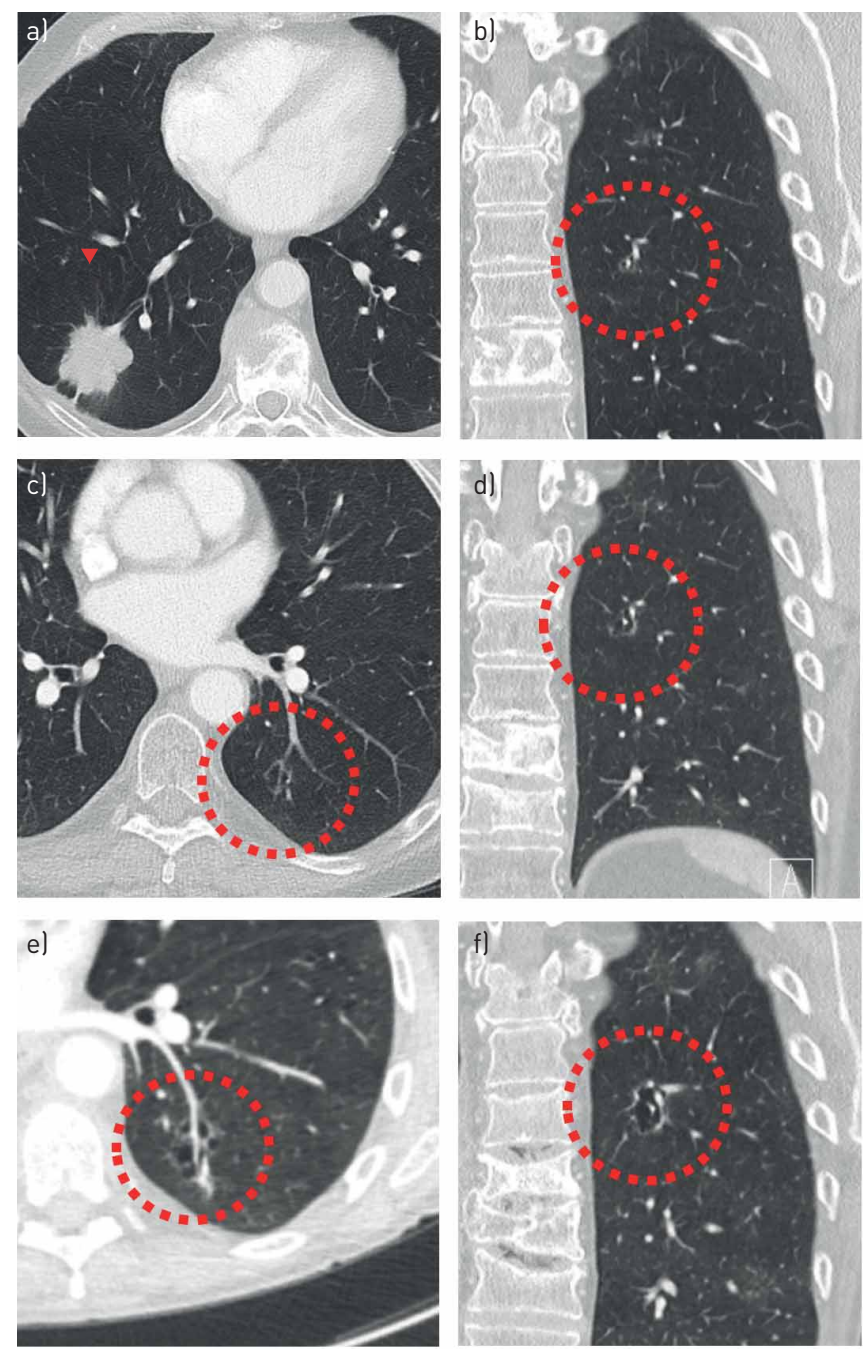

FIGURE 7 a-f) Increase in cystic airspace size, presumed to occur due to check-valve mechanism. A small cyst-related lesion in the left lower lobe (b) of a 64-year-old female with a history of breast carcinoma. This lesion was present at the time of diagnosis of a T2aN3M1b adenocarcinoma in the right lower lobe (a); however, it was not acknowledged. Over the course of 18 months the size and wall thickness progressed significantly (c, d, f) and eventually the lesion showed a solid component posteriorly (e). No pathology was obtained due to extensive comorbidity.

We have also encountered a substantial number of cases where cyst-related malignancies are metachronous or synchronous lung malignancies (figures 6 and 8), probably indicating a vulnerability of the lung tissue in these patients.

\section{Differential diagnosis}

Of course, not all cystic airspaces in relation to a solid component are malignant. There are several entities that can look like cyst-related lung malignancies (figure 9), which should be considered in the differential diagnosis. This includes cavitation of either infectious or malignant origin, especially tuberculosis and squamous cell carcinoma. Also, fungal infections can cause thick-walled cystic lesions with an endophytic nodule, as can rheumatoid arthritis. More rare mimickers, for example, are amyloid depositions, vasculitis and primary cystic metastases. Previous imaging, clinical information, patient history and ancillary findings often contribute significantly to assess lesion aetiology. For example, infection often progresses fast, may be multifocal and presents with clinical symptoms. In case of amyloid deposition, vasculitis, rheumatoid arthritis or cystic metastases, there is often multiplicity and guiding information in the patient history and/or additional clinical and laboratory findings that help to differentiate.

Nevertheless, it is not always possible to distinguish a primary cyst-related malignancy from a benign lesion (figure 10), and tissue is needed for a definite answer. Indication for invasive procedures or treatment should be decided on a case by case approach depending on comorbidities and patient 

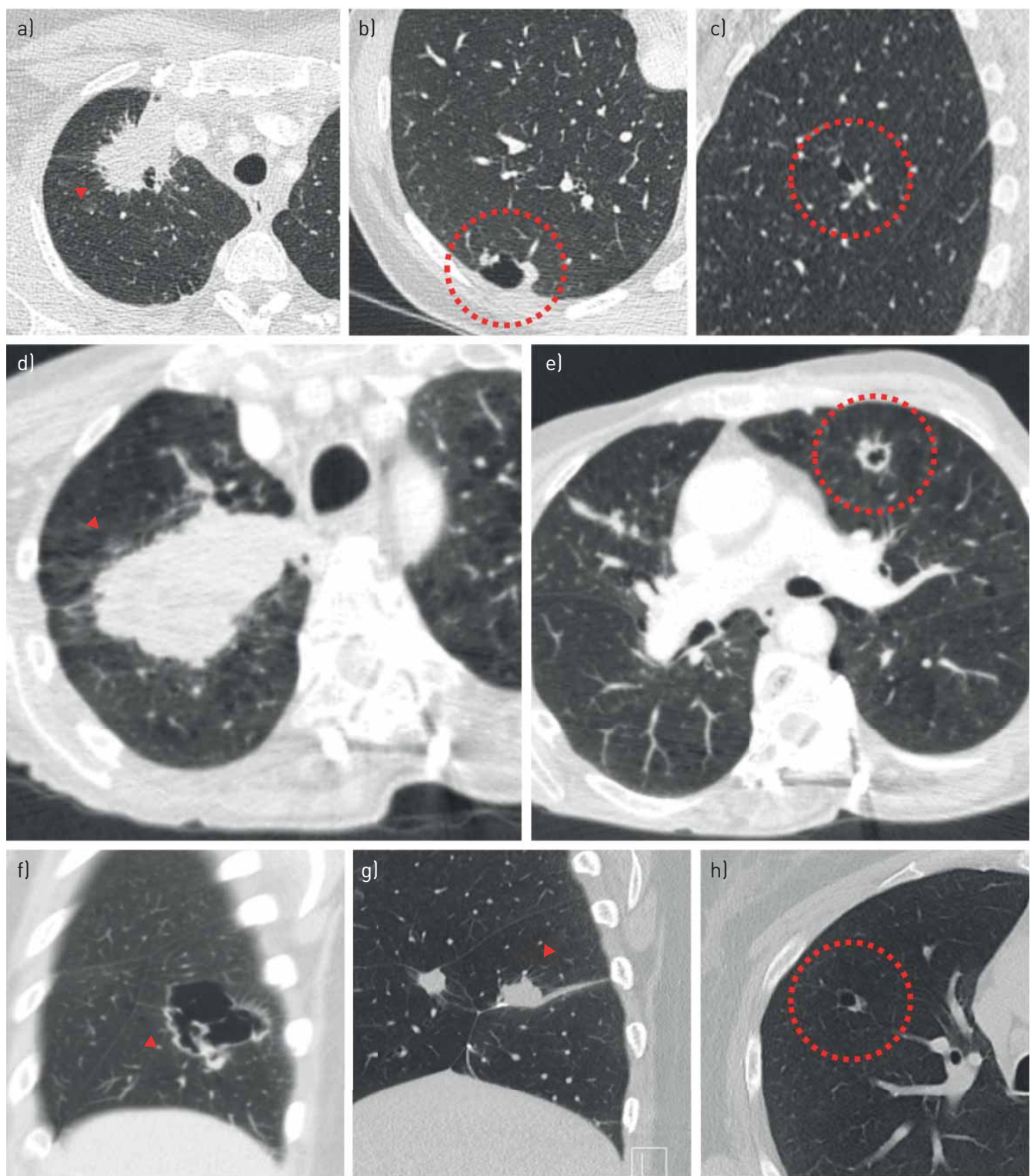

FIGURE 8 Cyst-related lung malignancies as metachronous or synchronous second primary lung cancers. a-e) Two patients with stage IV lung cancer with a large mass in the right upper lobe. Additional cyst-related lesions were present in other lung lobes. These lesions were persistent and highly suggestive of additional primary cyst-related lung malignancies; however, they were not pathologically proven due to the fact that results would not change treatment strategy in these patients. f) A third patient showed a large cystic airspace with irregular wall thickening in the right lower lobe, initially misinterpreted as a large cavitation with aspergillus. Perioperative frozen section showed a pleomorphic carcinoma in the right lower lobe. A metachronous solid nodule was present in the right middle (not shown). Only the solid component of the cyst-related lesion in the right lower lobe was resected, because neither lobectomy or bilobectomy was an option due to limited pulmonary capacity. gl After 1 year local recurrence was present together with persistent mass in the right middle lobe. h) A small cyst-related lesion in the right upper lobe was initially present, but, not acknowledged. This lesion showed an increase in wall thickness over time (not shown). All three lesions showed activity on a positron emission tomography-computed tomography scan obtained to confirm local recurrence.

preferences, in a multidisciplinary setting. Absolute cancer risk of lesions that morphologically conform to cyst-related lung malignancies is currently unknown, however, in our experience the vast majority represents (early) primary lung cancer.

\section{Clinical implications}

Flurodeoxyglucose-positron emission tomography computed tomography (FDG PET-CT) imaging may play a role in strengthening the suspicion of malignancy; however, is not able to distinguish primary lung 

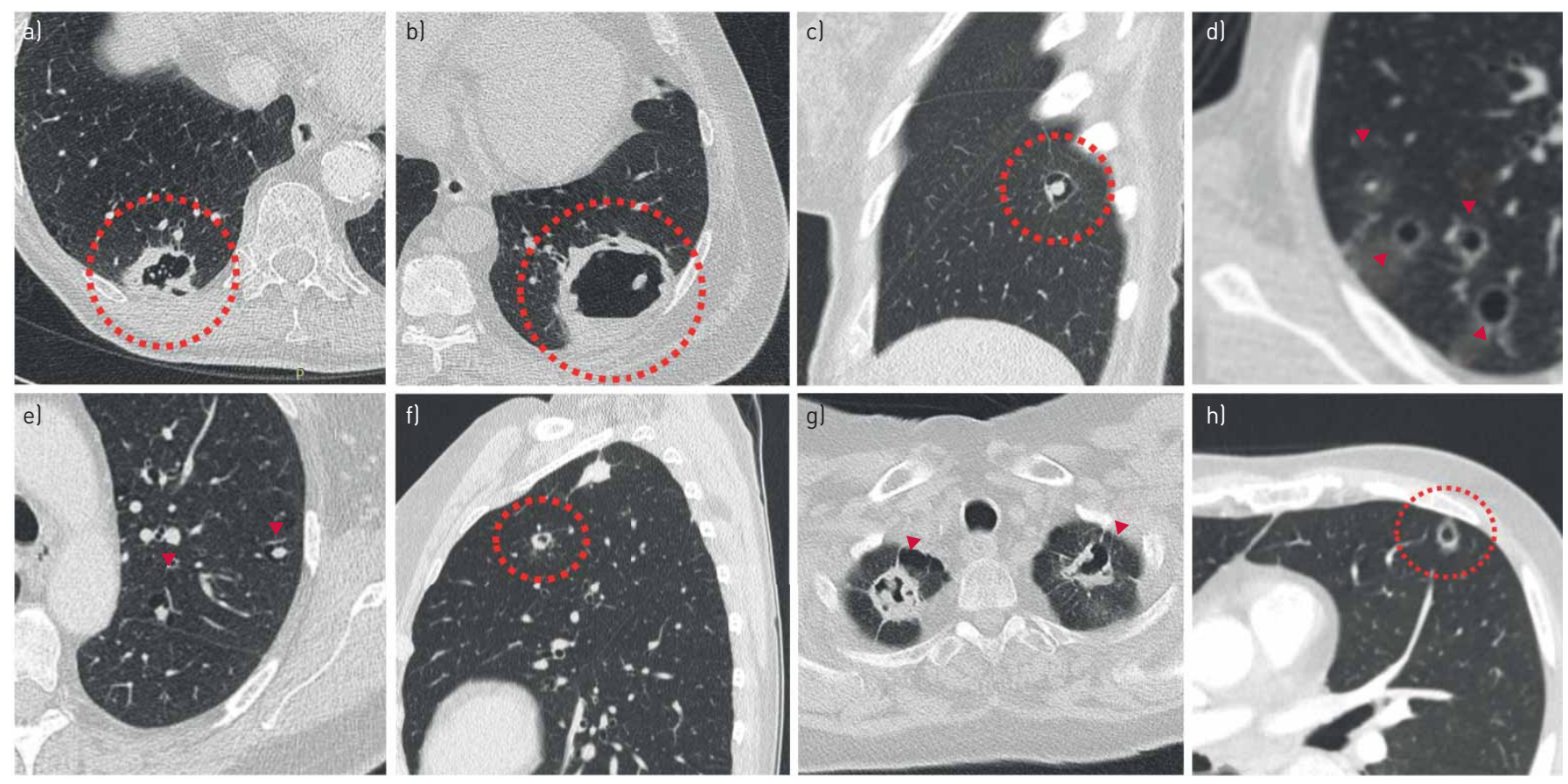

FIGURE 9 Lesions that represent potential mimickers of cyst-related primary lung malignancies. a) Irregular thick-walled cystic airspace in a 74-year-old male as an incidental finding on aortic imaging. Transthoracic biopsy showed chronic inflammatory changes without active components, and no signs of malignancy. The patient died 2 months later of an acute aortic rupture. b) One of several thick-walled cystic airspaces in a 62-year-old male with immunosuppression after kidney transplantation. Work-up showed invasive fungal infection, most likely mucormycosis. c) Thin-walled cystic airspace with an endophytic solid nodule, representing a rheumatoid nodule in a 60-year-old female with rheumatoid arthritis and several lung nodules. d) Several thin-walled cysts with ground-glass halo in a 73-year-old male with recurrent pneumothoraxes, first misinterpreted as infectious. After clinical deterioration and progression in size and number of cystic lung lesions pathology was obtained by wedge resection. It showed pure cystic metastases of a prior angiosarcoma of the scalp. The patient died shortly afterwards. e) Multiple thin-walled cysts with endo- and exophytic nodules in a 68-year-old female with Morbus-Sjögren and lymphoid interstitial pneumonia. Pathology showed that the solid components were amyloid depositions. f) Thick-walled cystic lesion in a 63-year-old female with allergic bronchopulmonary aspergillosis and aspergilloma. gl Multiple cavitating pulmonary nodules with a thick irregular wall in a 56-year-old female with vasculitis (granulomatosis with polyangiitis). h) Small subpleural thick-walled cyst in the left lower lobe of a 74-year-old male with a synchronous T1cN0M0 squamous cell carcinoma in the left upper lobe. Wedge resection was performed in addition to left upper lobe lobectomy, which showed chronic inflammatory changes without signs of a (second primary) malignancy.

cancer from infection or metastatic disease. More importantly, FDG PET-CT may also be misleading as not all cyst-related malignancies show FDG uptake [6-8]. Whether combined with FDG PET-CT or not, serial CT imaging is probably the best approach to evaluate changes in lesion aspect. One should focus on increase in size of the solid components and progressive solidification, as well as absence of response to
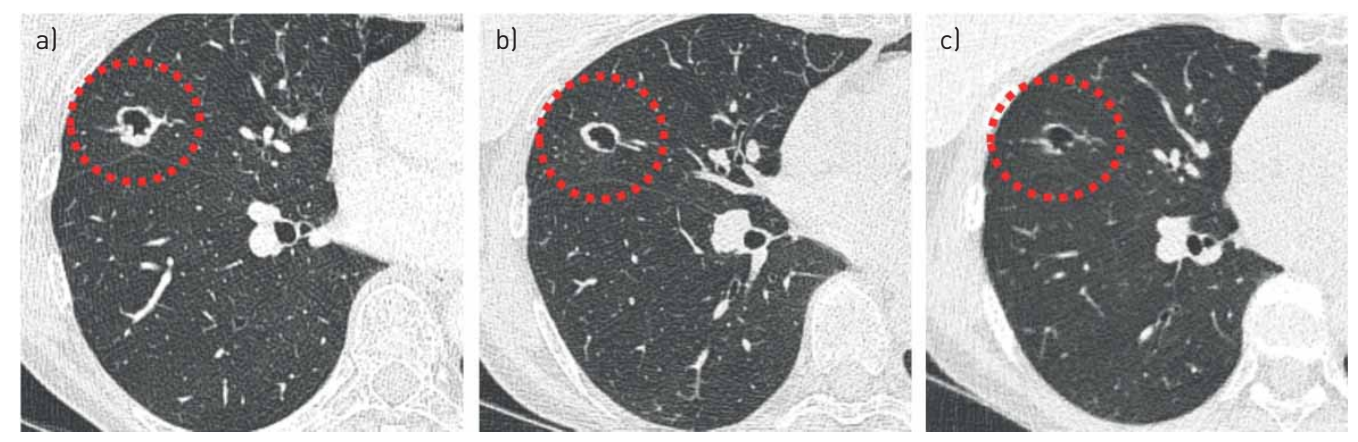

FIGURE 10 Highly suspected cyst-related lung lesion that proved to be of non-malignant aetiology. a) An irregular thick-walled cystic airspace in the right middle lobe of a 60-year-old female. The lesion was initially interpreted as a cavitating malignancy, presumably squamous cell carcinoma. Flurodeoxyglucose-positron emission tomography computed tomography (not shown) showed slight activity which was used to strengthen the suspicion for lung cancer. b) However, a pre-operative computed tomography scan showed decrease of the solid component, which changed the differential diagnosis towards infection. c) The decrease in wall thickness continued on serial imaging over a period of 8 months. The underlying microorganism remained unknown. 
anti-infectious therapy. All these findings point towards a malignant origin and should trigger work-up at an early stage to prevent delay (figures 11 and 12). Preferably, work-up includes obtaining tissue either from the lesion itself through a transthoracic biopsy or from lymph nodes or metastases, if present. However, although a pre-treatment histological diagnosis should be the default in the work-up of suspected malignancies, it is not always possible to obtain tissue. This is mainly due to extensive comorbidity, lesion location, expected complications or patient preferences. Also, it might be that biopsy will not alter management strategy and (wedge) resection covers both diagnosis and treatment. However, this mainly holds for the select group of cases with very high suspicion of malignancy and small lesions.

Current nodule management guidelines do not mention cyst-related malignancies as a separate entity, and due to a lack of prospective data there is no recommended and uniform surveillance strategy for these type of lesions. We believe that short-term imaging (3-6 months) to evaluate rapid changes is an important first step, given that there is evidence that cyst-related malignancies can be aggressive [5]. Short-term follow-up is also an important strategy to re-evaluate lesions in case there is doubt as to whether a real
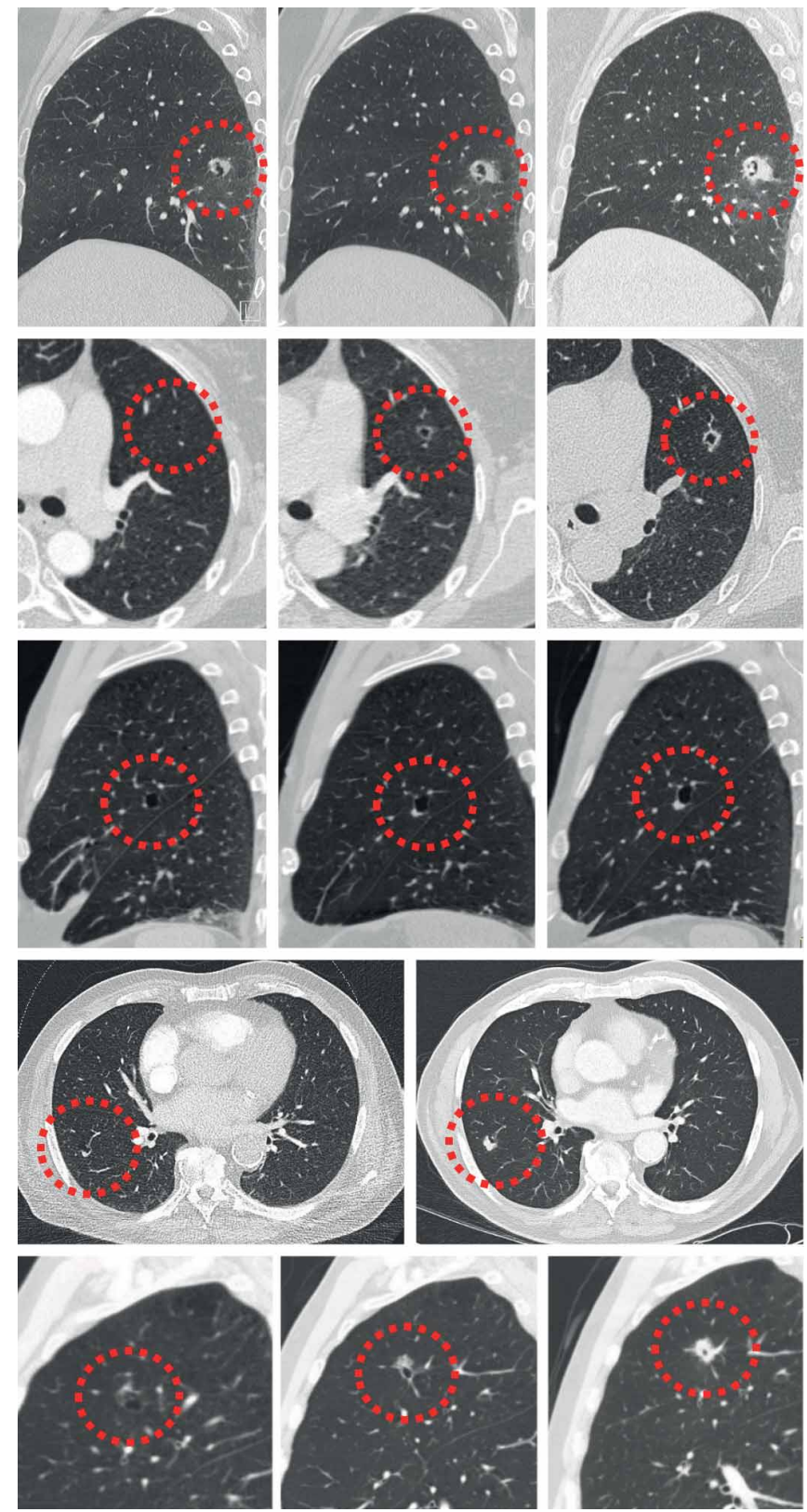

FIGURE 11 Evolution of cyst-related primary lung malignancies. Five different cyst-related primary lung malignancies showing increase in solid components and progressive solidification over time linterval between 5 months and 2.5 years). These are signs of a malignant aetiology that should trigger appropriate action. 

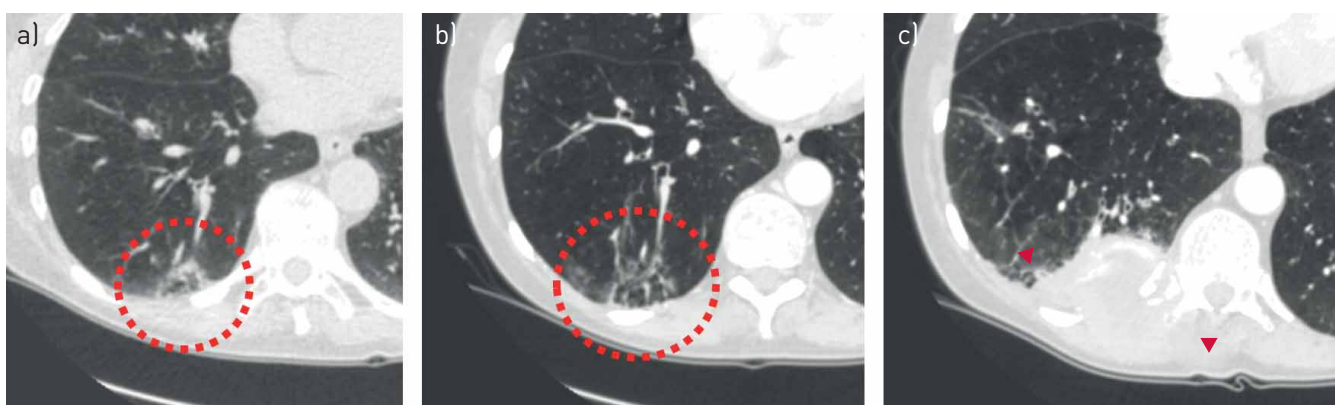

FIGURE 12 A change of lesion aspect should trigger action. a) A multilocular cystic lesion in the right lower lobe of a 67-year-old female was interpreted as nonspecific. b) Its aspect changed over 6 months with increase in total size, more cystic and solid components as well as some ground glass, which did not trigger appropriate action. c) The lesion eventually evolved into a large solid adenocarcinoma with invasion of the chest wall 2 years later. The patient died shortly after due to metastatic disease.

cyst-related lesion is encountered. In general, persistent and progressive cyst-related lesions should be differentiated from irrelevant interstitial thickening in emphysematous airspaces and other smoking-related and infectious lung changes.

Until more data becomes available cyst-related lesions may be followed after exclusion of fast growth, similar to suspected (sub)solid lung nodules. However, we feel it is reasonable and good clinical practice to intervene as soon as the aspect of the lesion changes and/or when targetable solid components are present, especially in patients without substantial comorbidity and with substantial life expectancy. Currently, too little is known on whether close surveillance might be an alternative for invasive intervention, as has been shown for subsolid lesions [12].

Besides the fact that the optimal follow-up strategy for cyst-related lung lesions is unknown, measuring and staging is also not standardised. Regarding measurements one may propose to use the maximum diameter of the focal solid component and ignore the cystic part. However, total lesion size including the cystic airspace may be more suitable when (near) circumferential cyst wall thickening is present. Inevitably, measuring this morphologically highly variable group of lung malignancies is difficult and results are prone to significant inter-reader variability.

\section{Future directions}

As available data is still limited, future (prospective) studies should focus on answering the currently remaining questions on frequency and absolute cancer risk in clinical and screening cohorts, in relation to patient characteristics. In addition, differences in biological behaviour and prognostic value of imaging subtypes of cyst-related malignancies should be unravelled, and measurements and staging will benefit from standardisation.

\section{Conclusion}

It is increasingly recognised that (early) lung cancer sometimes presents as cyst-related lesions on CT imaging, which may often remain undetected and misinterpreted. This could lead to serious delay in diagnosis and associated burden. Therefore, increased awareness of this atypical lung cancer morphology is urgently needed among both radiologists and clinicians. In cases with lack of clinical or radiological characteristics pointing towards an obvious benign differential diagnosis, all cyst-related lesions should be interpreted as suspicious for primary lung cancer and managed accordingly.

Acknowledgements: The authors would like to thank the radiology departments of Amsterdam University Medical Centre (Amsterdam, The Netherlands) and St. Antonius Hospital (Nieuwegein, The Netherlands) for the use of images originating from their patient population.

Conflict of interest: O.M. Mets reports personal fees (as an expert panel member) from Aidence, outside the submitted work. C.M. Schaefer-Prokop has nothing to disclose. P.A. de Jong has nothing to disclose.

Support statement: This work was supported by the Dutch Cancer Foundation (grant number KUN-2013-6110). Funding information for this article has been deposited with the Crossref Funder Registry.

\section{References}

1 MacMahon H, Naidich DP, Goo JM, et al. Guidelines for management of incidental pulmonary nodules detected on CT images: from the Fleischner Society 2017. Radiology 2017; 284: 228-243.

2 Callister ME, Baldwin DR, Akram AR, et al. British Thoracic Society guidelines for the investigation and management of pulmonary nodules. Thorax 2015; 70: Suppl. 2, ii1-ii54. 
3 Henschke CI, Yankelevitz DF, Mirtcheva R, et al. CT screening for lung cancer: frequency and significance of part-solid and nonsolid nodules. AJR Am J Roentgenol 2002; 178: 1053-1057.

4 Hasegawa M, Sone S, Takashima S, et al. Growth rate of small lung cancers detected on mass CT screening. Br J Radiol 2000; 73: 1252-1259.

5 Farooqi AO, Cham M, Zhang L, et al. Lung cancer associated with cystic airspaces. AJR Am J Roentgenol 2012; 199: 781-786.

6 Mascalchi M, Attinà D, Bertelli E, et al. Lung cancer associated with cystic airspaces. J Comput Assist Tomogr 2015; 39: 102-108.

7 Fintelmann FJ, Brinkmann JK, Jeck WR, et al. Lung cancers associated with cystic airspaces: natural history, pathologic correlation, and mutational analysis. J Thorac Imaging 2017; 32: 176-188.

8 Guo J, Liang C, Sun Y, et al. Lung cancer presenting as thin-walled cysts: an analysis of 15 cases and review of literature. Asia Pac J Clin Oncol 2016; 12: e105-e112.

9 Scholten ET, Horeweg N, de Koning HJ, et al. Computed tomographic characteristics of interval and post screen carcinomas in lung cancer screening. Eur Radiol 2015; 25: 81-88.

10 Sheard S, Moser J, Sayer C, et al. Lung cancers associated with cystic airspaces: underrecognized features of early disease. Radiographics 2018; 38: 704-717.

11 Maki D, Takahashi M, Murata K, et al. Computed tomography appearances of bronchogenic carcinoma associated with bullous lung disease. J Comput Assist Tomogr 2006; 30: 447-452.

12 Scholten ET, de Jong PA, de Hoop B, et al. Towards a close computed tomography monitoring approach for screen detected subsolid pulmonary nodules? Eur Respir J 2015; 45: 765-773. 